Zelasqui, I.O. Más allá de una problemática urbana: Accesibilidad edilicia y educación inclusiva para personas con discapacidad. Derecho y Ciencias Sociales. Mayo -Octubre 2021. № 25 .Pgs 100-122. ISSN 1852-2971. Instituto de Cultura Jurídica y Maestría en Sociología Jurídica. Facultad de Ciencias Jurídicas y Sociales. Universidad Nacional de La Plata. Argentina.

\title{
Más allá de una problemática urbana: Accesibilidad edilicia y educación inclusiva para personas con discapacidad
}

Beyond an urban problem:Building accessibility and inclusive education for people with disabilities

Ignacio Oscar Zelasqui $\bullet$

\section{Resumen}

Este trabajo tiene por objeto la problemática de accesibilidad a la educación por parte de personas con discapacidad. Se enfoca en la dimensión arquitectónica o edilicia, en tanto otros aspectos del acceso a la educación no son abordados en este artículo. Se propone, por un lado, describir la situación de las escuelas en la ciudad de La Plata en materia de accesibilidad edilicia y estándares de derechos humanos, tomando como punto de partida un relevamiento llevado a cabo en los años 2012/2013 por la Clínica Jurídica de Derechos Humanos y Discapacidad, programa de extensión perteneciente a la Facultad de Ciencias Jurídicas y Sociales (UNLP). Por otra parte, presentar diversas dimensiones y posibles herramientas analíticas para pensar la problemática de accesibilidad a la educación inclusiva para personas con discapacidad. En consecuencia, se exhibirán algunas relaciones de esta problemática con otros ejes o procesos tales como movimientos sociales e identidades, espacios y políticas públicas, los cuales abren múltiples interrogantes y líneas preliminares de análisis. Se trata de la disputa por el espacio material, sus interpretaciones y representaciones. La accesibilidad física o edilicia significa entonces una lucha también por una reinterpretación del espacio escuela y la educación. Las corporalidades disidentes en las escuelas ponen de relieve tensiones y contradicciones de ese espacio normalizado y permiten (re)pensar nuevos espacios que faciliten otros modos de vinculación y pedagogía, en este caso, inclusivos en la diversidad.

Palabras clave: Discapacidad ; Accesibilidad ; Derechos Humanos ; Problemas urbanos

\begin{abstract}
This work is aimed at the problem of accessibility to education for people with disabilities. It focuses on the architectural or building dimension, while other aspects of access to education are not addressed in this article. It is proposed, on the one hand, to describe the situation of schools in the city of La Plata in terms of building accessibility and human rights standards, taking as a starting point a survey carried out in 2012/2013 by the Legal Clinic of Human Rights and Disability, an extension program belonging to the Faculty of Legal and Social Sciences (UNLP). On the other hand, present various dimensions and possible analytical tools to think

\footnotetext{
- Abogado (FCJyS-UNLP). Becario investigador de la Universidad Nacional de La Plata (FCJyS-GECSI). Cursando la carrera de Doctorado en Ciencias Sociales (FaHCE-UNLP). Docente adscripto en Sociología Jurídica Cátedra I (FCJyS-UNLP). Integrante de Clínica Jurídicas Comisión Discapacidad y Derechos Humanos (FCJySUNLP). Correo: izelasqui@jursoc.unlp.edu.ar
} 
Zelasqui, I.O. Más allá de una problemática urbana: Accesibilidad edilicia y educación inclusiva para personas con discapacidad. Derecho y Ciencias Sociales. Mayo -Octubre 2021. № 25 .Pgs 100-122. ISSN 1852-2971. Instituto de Cultura Jurídica y Maestría en Sociología Jurídica. Facultad de Ciencias Jurídicas y Sociales. Universidad Nacional de La Plata. Argentina.

about the problem of accessibility to inclusive education for people with disabilities. Consequently, some relationships of this problem with other axes or processes such as social movements and identities, spaces and public policies will be exhibited, which open multiple questions and preliminary lines of analysis. It is about the dispute over material space, its interpretations and representations. Physical or building accessibility then means a struggle also for a reinterpretation of the school space and education. Dissident corporalities in schools highlight tensions and contradictions of this normalized space and allow (re) thinking of new spaces that facilitate other ways of linking and pedagogy, in this case, inclusive of diversity.

Keywords: Disability ; Accessibility ; Human Rights ; Urban problems 
Zelasqui, I.O. Más allá de una problemática urbana: Accesibilidad edilicia y educación inclusiva para personas con discapacidad. Derecho y Ciencias Sociales. Mayo -Octubre 2021. № 25 .Pgs 100-122. ISSN 1852-2971. Instituto de Cultura Jurídica y Maestría en Sociología Jurídica. Facultad de Ciencias Jurídicas y Sociales. Universidad Nacional de La Plata. Argentina.

\section{Más allá de una problemática urbana: Accesibilidad edilicia y educación inclusiva para personas con discapacidad}

Ignacio Oscar Zelasqui

\section{Introducción.}

La accesibilidad edilicia en establecimientos educativos se vincula estrechamente, aunque no agota, al reconocimiento efectivo en el derecho a la educación inclusiva para personas con discapacidad. Bajo esta premisa, la Clínica Jurídica de Derechos Humanos y Discapacidad, programa de extensión perteneciente a la Facultad de Ciencias Jurídicas y Sociales (UNLP), realizó un relevamiento en los años 2012/2013 en establecimientos educativos de la ciudad de La Plata. Los resultados obtenidos en dicha muestra fueron el sustento fáctico principal de la acción judicial de alcance colectivo iniciada a finales del año 2015 contra Gobierno de la Provincia de Buenos Aires y su Dirección General de Cultura y Educación, causa que continúa en trámite en la actualidad ante el Juzgado Contencioso Administrativo $\mathrm{N}^{\circ} 2$ de La Plata caratulada "FUNDACIÓN ACCESO YA C/ DIRECCIÓN GENERAL DE CULTURA Y EDUCACION S/ PRETENSION RESTABLECIMIENTO O RECONOC. DE DERECHOS OT”. En este proceso se evidenció la situación de las escuelas en materia de accesibilidad, poniendo de relieve las políticas públicas llevadas a cabo por la provincia en este sentido y su correspondencia con los estándares de derechos humanos que la obligan.

Entendiendo que esta problemática no se agota en el reconocimiento judicial de un derecho humano fundamental para las personas con discapacidad, es pertinente reflexionar sobre el lugar "escuelas", los procesos de construcción de esos lugares, sus efectos respecto de las personas, los cuerpos y los capitales y bienes en disputa. A su vez, la estrategia de intervención mediante la Clínica Jurídica permite problematizar la formulación tradicional de políticas públicas, facilitando ámbitos de participación y la transformación de dicho espacio en el marco de un litigio de carácter estructural.

\section{Algunos lineamientos metodológicos:}

El abordaje se focalizó en caracterizar las escuelas objeto de estudio a través del cruce de diferentes variables referenciándolas con la normativa vigente sobre accesibilidad arquitectónica para personas con discapacidad. Para relevar las condiciones edilicias de accesibilidad consideramos 5 variables: la existencia de rampas, altura de timbre, baño adaptado, amplitud y circulación en aulas y patio. Se confeccionaron planillas numeradas por 
Zelasqui, I.O. Más allá de una problemática urbana: Accesibilidad edilicia y educación inclusiva para personas con discapacidad. Derecho y Ciencias Sociales. Mayo -Octubre 2021. № 25 .Pgs 100-122. ISSN 1852-2971. Instituto de Cultura Jurídica y Maestría en Sociología Jurídica. Facultad de Ciencias Jurídicas y Sociales. Universidad Nacional de La Plata. Argentina.

cada escuela relevada en la que consten los datos del establecimiento (nombre y dirección) y una descripción de cada variable donde se consigna su accesibilidad o no. Finalmente se realizó una tabla con los resultados totales que contiene 5 columnas: nombre del establecimiento, número de planilla correspondiente, dirección, accesibilidad (si o no) y observaciones (en que variables y por qué no cumple, más otros datos de relevancia).

Durante el monitoreo se revelaron algunos problemas metodológicos ya que algunas autoridades de las escuelas, pese a tener permiso firmado por la Inspectora Jefe Distrital de La Plata, se negaron a permitir el acceso al edificio dificultando el relevamiento de todas las variables previstas. En consecuencia, consideramos escuela accesible aquella que cumple con estas cinco variables. En el mismo sentido, entendemos por escuela no accesible aquella que habiendo observado todas las variables no cumple con alguna de ellas. Con esta categorización y ante las limitaciones mencionadas, clasificamos los resultados en tres: Escuelas accesibles, no accesibles y sin determinar (porque no se pudo medir alguna de las variables que consideramos para definir la categoría).

El proceso de recolección se realizó entre los años 2012 y 2013 e incluyó a varios actores sociales: integrantes de la Clínica Jurídica de Discapacidad y Derechos Humanos (graduados y estudiantes de derecho), estudiantes de otras carreras (comunicación social y ciencias de la educación), personas con discapacidad, organizaciones sociales y movimientos de PCD (Acceso ya, Asosiacion azul, entre otras) y difusion mediatica.

La muestra estuvo compuesta por 154 establecimientos educativos de la ciudad de La Plata que representan más de un tercio del total (poco más de 400 establecimientos educativos, esto es jardines, escuelas primaria y secundaria públicas y privadas, "normales" y "especiales" de la ciudad de La Plata al momento de realizar el relevamiento).

\section{Marco jurídico constitucional.}

I) Obligaciones del Estado y derechos de las personas con discapacidad:

En primer término, es menester conceptualizar los derechos humanos que se ponen en juego cuando hablamos de accesibilidad o barreras arquitectónicas en edificios públicos o privados y las obligaciones que tiene el Estado en dicho marco jurídico conceptual. Obligaciones y derechos que se agudizan al hablar de un grupo en situación de vulnerabilidad con protección preferencial y cuando se trata a su vez, como el supuesto en estudio, de edificios educativos. 
Zelasqui, I.O. Más allá de una problemática urbana: Accesibilidad edilicia y educación inclusiva para personas con discapacidad. Derecho y Ciencias Sociales. Mayo -Octubre 2021. № 25 .Pgs 100-122. ISSN 1852-2971. Instituto de Cultura Jurídica y Maestría en Sociología Jurídica. Facultad de Ciencias Jurídicas y Sociales. Universidad Nacional de La Plata. Argentina.

El plexo normativo que describiremos se funda especialmente en la letra, espíritu y principios liminares de la "Convención Internacional Sobre los Derechos de las Personas con Discapacidad" (adoptada por la Asamblea General de ONU el 13 de diciembre de 2006, aprobada por nuestro país el 21 de mayo de 2008 mediante la Ley 26.378 y dotada de rango constitucional desde el 19 de noviembre de 2014 por medio de la ley 27.044), primer tratado de derechos humanos del siglo XXI. Nos referimos a sus postulados de Diseño Universal, Ajustes Razonables y Educación Inclusiva, en el paradigma o modelo social ${ }^{1}$ de la discapacidad así como en la importancia de la participación de las personas con discapacidad en las políticas que los afectan directamente y en el rol de las organizaciones que los representan; así como en la "Convención Interamericana para la Eliminación de Todas las Formas de Discriminación contra las Personas con Discapacidad" (aprobada con fecha 6 de julio de 2000 mediante la Ley 25.280 y ratificada el 10 de enero de 2001).

La República Argentina como Estado Parte ha asumido el compromiso de adoptar medidas para eliminar los obstáculos arquitectónicos, de transporte y comunicaciones que existan, con la finalidad de facilitar el acceso y uso para las personas con discapacidad, y posee la obligación de desarrollar medidas legislativas, sociales, educativas, laborales y de cualquier otra índole a fin de eliminar cualquier tipo de discriminación hacia las personas con discapacidad y propiciar su plena integración en la sociedad.

En el año 1994 el Estado Nacional sancionó la Ley 24.314 que modifica la Ley 22.431 (“Sistema de Protección Integral de los discapacitados”), cuyo art. 20 establece: "... la prioridad

\footnotetext{
${ }^{1}$ Respecto de los orígenes y características del modelo social de la discapacidad plasmado luego en la Convención, se inicia con las demandas y politización del movimiento social de las personas con discapacidad a partir de la década de 1960 en diversas partes del mundo, cuyos primeros desarrollados académicos se dieron en EEUU y el Reino Unido (Mike Oliver). Esta corriente entiende que las causas que originan la discapacidad no son ni religiosas, ni científicas, sino sociales o al menos, preponderantemente sociales. Hasta ese momento los modelos de prescindencia y médico rehabilitadores habían considerado que las personas con discapacidad eran innecesarias o desechables. Más allá del extremo eugenésico, en la prescindencia el abordaje se centró principalmente en la segregación, se las trató de aislar y ocultar en el ámbito familiar o en instituciones creadas a dichos fines haciéndolas depender permanentemente de su familia o la asistencia del estado. Con el modelo médico se considera que la persona puede ser "útil" o valiosa socialmente e integrarse siempre que sea "curada" o rehabilitada para alcanzar los criterios de normalidad impuestos socialmente.

Para el modelo social la discapacidad es un proceso determinado por componentes históricos, socioeconómicos, culturales, biológicos y psicológicos. Las características de la persona tienen importancia en la medida en que evidencian la capacidad -o incapacidad- de la sociedad para dar respuesta a sus necesidades: es la propia sociedad la que discapacita y excluye del ejercicio de los derechos. De este modo, se realiza una distinción entre lo que comúnmente se denomina deficiencia (diversidad funcional) y lo que se entiende por discapacidad. El desafío reside en un "qué" hacer por parte de la sociedad y del Estado, enfocado en remover barreras y prejuicios, que es donde nace y se consolida la exclusión y subestimación de la PCD. A modo de ejemplo, una persona que en su diversidad funcional se desplaza en silla de ruedas no es una persona con discapacidad hasta que encuentra una $\underline{\text { barrera, }}$, como la falta de rampas, que no le permiten acceder a determinados derechos, servicios, intereses y deseos.
} 
Zelasqui, I.O. Más allá de una problemática urbana: Accesibilidad edilicia y educación inclusiva para personas con discapacidad. Derecho y Ciencias Sociales. Mayo -Octubre 2021. № 25 .Pgs 100-122. ISSN 1852-2971. Instituto de Cultura Jurídica y Maestría en Sociología Jurídica. Facultad de Ciencias Jurídicas y Sociales. Universidad Nacional de La Plata. Argentina.

de la supresión de barreras físicas en los ámbitos urbanos arquitectónicos y del transporte que se realicen o en los existentes que remodelen o sustituyan en forma total o parcial sus elementos constitutivos con el fin de lograr la accesibilidad para las personas con movilidad reducida ....". Con miras a tal fin la misma ley invita a las provincias a adherir y/o incorporar en sus respectivas normativas los contenidos de la presente.

En el ámbito provincial, la Ley 10.592 que regula el Régimen Jurídico Básico e Integral para las Personas Discapacitadas, establece que el Estado provincial “(...) asegurará los servicios de atención médica, educativa (...). Asimismo, brindará los beneficios y estímulos que permitan neutralizar su discapacidad,(...) y procurará eliminar las desventajas que impidan una adecuada integración familiar, social, cultural, económica, educacional y laboral...”. En especial, el artículo 24 dispone: “Todo edificio de organismo público o privado que se proyecte en el futuro y cuyo destino implique el uso del mismo por la población en general, deberá prever accesos, medios de circulación interna e instalaciones de servicio que permitan su utilización por personas discapacitadas (...)".

En consecuencia, el derecho a la accesibilidad física de las personas con discapacidad se encuentra reconocido y garantizado en diversos cuerpos normativos, tanto a nivel nacional como internacional, tratándose de una garantía constitucional que tiene como eje fundamental la igualdad real -no meramente declarada- entre las personas.

II) Fundamentos jurídicos que obligan a la administración pública provincial a realizar las adaptaciones necesarias y los derechos en peligro frente a la omisión estatal:

A) La violación del derecho a un trato igualitario y el principio de no discriminación.

La igualdad como interés colectivo y principio rector del estado democrático de derecho encuentra recepción constitucional a través del principio de igualdad (Art. 16, 20 C.N), de las acciones afirmativas (Art. 37, 75. Inc.19, 75. Inc.23 C.N) y del principio de no discriminación (Art. 75. Inc. 22 C.N, Declaración Americana de los Derechos y Deberes del Hombre Art. II; de la Declaración Universal de Derechos Humanos Art. 7; de la Convención Americana sobre Derechos Humanos Art. 24, 1; del Pacto Internacional de Derechos Civiles y Políticos Art. 26 y del Pacto Internacional de Derechos Económicos, Sociales y Culturales Art. 2).

Dicha vocación igualitaria no sólo proclama una igualdad ante la ley o formal sino también sustancial y fáctica. En relación a la protección de grupos sociales en situación de vulnerabilidad, el programa constitucional igualitario se traduce en una obligación de igual consideración y respeto consistente en la eliminación de obstáculos y en la dotación de recursos 
Zelasqui, I.O. Más allá de una problemática urbana: Accesibilidad edilicia y educación inclusiva para personas con discapacidad. Derecho y Ciencias Sociales. Mayo -Octubre 2021. № 25 .Pgs 100-122. ISSN 1852-2971. Instituto de Cultura Jurídica y Maestría en Sociología Jurídica. Facultad de Ciencias Jurídicas y Sociales. Universidad Nacional de La Plata. Argentina.

para la igualdad de oportunidades. En relación a las personas con discapacidad, esto último se desprende de su inclusión dentro del artículo 75 Inc. 23 de la C.N como así también del texto de la Convención Internacional sobre los Derechos de las Personas con Discapacidad.

Además, en la legislación infra constitucional, tanto a nivel nacional como provincial, existe una vasta regulación específica en punto a la accesibilidad al medio físico para personas con movilidad reducida, consagrando la obligación de supresión de barreras “físicas”, en las vías y espacios públicos y "arquitectónicos”, en los edificios de uso público, sean de propiedad pública o privada. Por motivos de extensión y evitando la reiteración de artículos con regulación similar, resalto que incluso la ley de educación provincial contempla previsiones al respecto (arts. 5 y 8 de la ley 13.688) y se ha llegado a reglamentar minuciosamente los aspectos arquitectónicos necesarios para cumplir con la accesibilidad en los edificios (decreto 1149/90).

B) La violación del derecho a la educación inclusiva de niños, niñas y adolescentes con discapacidad como mecanismo de segregación social.

El derecho a la educación resulta valioso en sí mismo al comprender el derecho a aprender y a compartir un espacio de inclusión e interacción social y de vida en comunidad. A la vez reviste un carácter instrumental, al erigirse como precondición de otros derechos; en especial la relación entre el derecho a la educación, el trabajo, la cultura y el medio social, al incidir sobre la formación para el futuro y facilitar la autonomía posibilitando el diseño del propio plan de vida. Por otro lado cobra significación democrática, entendida esta como un espacio deliberativo entre sujetos iguales, por constituir la escuela un espacio central en la construcción de ciudadanía. Por esto las desigualdades fácticas como la obstaculización del derecho a la educación repercuten negativamente en la participación y deliberación política de todo un colectivo así como en el acceso al desarrollo laboral.

En este sentido entonces, es posible identificar ciertos obstáculos para el acceso a la educación inclusiva como mecanismos de segregación sistemática (entendida ésta como la existencia de estructuras y prácticas sociales que obstaculizan el desarrollo de las libertades). En esta línea, la no eliminación de obstáculos al ejercicio del derecho a la educación constituye una faceta de la opresión por marginación, es decir, por un proceso de exclusión de determinados grupos de la participación útil y plena en la sociedad. Cabe resaltar que para que esto suceda no es necesaria la existencia de una institucionalidad formal de segregación, ni una voluntad explícita, sino que basta con formas más sutiles o escondidas, o con advertir, sencillamente, sus efectos en la vida cotidiana de esta población. Así mismo, toda esta estructura de opresión 
Zelasqui, I.O. Más allá de una problemática urbana: Accesibilidad edilicia y educación inclusiva para personas con discapacidad. Derecho y Ciencias Sociales. Mayo -Octubre 2021. № 25 .Pgs 100-122. ISSN 1852-2971. Instituto de Cultura Jurídica y Maestría en Sociología Jurídica. Facultad de Ciencias Jurídicas y Sociales. Universidad Nacional de La Plata. Argentina.

desemboca, inexorablemente, en una marginación futura por carencia de poder y libertad de todo un grupo social, ya que es dable pensar que quien no cuenta con aquellos "insumos" culturales tendrá menos poder social para reclamar sus derechos, participar en las decisiones políticas y gozar de cierta autonomía laboral. En efecto, las personas que carecen de poder son siempre receptoras del ejercicio de poder de otras pero casi nunca ejercen el propio. Este tipo de opresión cultural -y simbólica- se cristaliza en la medida en que los grupos sociales dominantes definen, promueven y universalizan las pautas sociales que deben ser consideradas "normales" o "legítimas" al tiempo que definen, caracterizan y estereotipan a los grupos con menos poder de manera disvaliosa (Disidencias Sexuales/Promiscuos; Migrantes/Narcotraficantes; Personas con Discapacidades/Incapaces, etc.) asignando lugares y roles sociales subalternos.

En materia de educación de personas con discapacidad el modelo social se traduce en el derecho humano a la educación inclusiva. La Declaración de Salamanca y Marco de Acción para las Necesidades Educativas Especiales y la Convención Sobre los Derechos de las Personas con Discapacidad en su artículo 24 receptan de manera vigorosa el derecho a la educación inclusiva entendido como un sistema educativo capaz de adaptarse, recibir y brindar educación a todos los niños y niñas en establecimiento comunes, por tanto no es aceptable una política que solo garantice la accesibilidad de las escuelas especiales ya que dicha política confronta directamente con la letra y el espíritu de la Convención y de la jurisprudencia internacional.

Es evidente que para alcanzar los postulados internacionales de los que Argentina es parte y se ha obligado a cumplir, se hace necesario detectar y eliminar los diferentes obstáculos a través de la recopilación de información y la adopción de medidas de acción dirigidas a ese fin, entre ellas la eliminación de barreras arquitectónicas.

\section{Proceso de alcance colectivo con perfil "estructural": Las escuelas en La Plata.}

Atento reiteradas denuncias y manifestación recibida por personas con discapacidad que acuden y participan de la Clínica Jurídica de Derechos Humanos y Discapacidad (en adelante, Clínica Jurídica), programa de extensión perteneciente a la Facultad de Ciencias Jurídicas y Sociales (UNLP), dicho espacio se propuso realizar un relevamiento para tomar conocimiento sobre el estado de las escuelas de la ciudad de La Plata en materia de accesibilidad. Los resultados del mismo se constituyeron en el sustento fáctico principal de la posterior demanda con alcance colectivo iniciada a finales del año 2015 contra Gobierno de la Provincia de Buenos Aires y su Dirección General de Cultura y Educación, causa que continúa en trámite en la 
Zelasqui, I.O. Más allá de una problemática urbana: Accesibilidad edilicia y educación inclusiva para personas con discapacidad. Derecho y Ciencias Sociales. Mayo -Octubre 2021. № 25 .Pgs 100-122. ISSN 1852-2971. Instituto de Cultura Jurídica y Maestría en Sociología Jurídica. Facultad de Ciencias Jurídicas y Sociales. Universidad Nacional de La Plata. Argentina.

actualidad, ante el juzgado contencioso administrativo $\mathrm{N}^{\circ} 2$ de La Plata caratulada "FUNDACIÓN ACCESO YA C/ DIRECCIÓN GENERAL DE CULTURA Y EDUCACION S/ PRETENSION RESTABLECIMIENTO O RECONOC. DE DERECHOS - OTROS JUCIO”. Dicha demanda tiene por objeto se ordene implementar las medidas necesarias para garantizar la accesibilidad edilicia y arquitectónica adecuada a los niños, niñas y adolescentes con discapacidad motriz a la totalidad de las escuelas públicas y privadas de la Ciudad de La Plata.

Este pedido se funda en que las escuelas públicas y privadas de la ciudad, que por mandato constitucional y convencional deberían poder recibir a todas las personas con discapacidad, no pueden hacerlo porque en su mayoría son inaccesibles. La Clínica Jurídica ha llegado a esta conclusión luego de haber realizado un pormenorizado estudio de campo a través de la recolección y medición de datos.

El equipo de trabajo de la Clínica se abocó a lo largo del año 2012/2013, a comprobar si las condiciones mínimas de infraestructura y seguridad garantizaban la igualdad educativa para alumnos/as con algún tipo de discapacidad motriz y verificar el grado de cumplimiento de la normativa vigente en punto a accesibilidad al medio físico por parte de PCD. Posteriormente se emprendió un relevamiento que lejos de arrojar resultados positivos, comprobó un alto grado de incumplimiento.

El emprendimiento consistió en el relevamiento de condiciones edilicias de accesibilidad, entre ellas: la existencia de rampas, baños adaptados, amplitud de circulación, altura de timbres, etc., en vista de determinar su ajuste a las normas que regulan la materia en forma minuciosa. La confección de esta prueba con perfil estructural requirió, a su vez, de mayores recursos humanos, una mejor organización en red (estudiantes de otras carreras, difusión mediática, colaboración de varias organizaciones sociales y movimientos de PCD) como también, una mayor cooperación dentro del ámbito académico.

El resultado del trabajo de campo evidencia un panorama poco alentador: un altísimo porcentaje de incumplimiento normativo en lo referente a supresión de barreras y accesibilidad al medio físico. De los poco más de 400 establecimientos educativos, esto es jardines, escuelas primaria y secundaria públicas y privadas, "normales" y "especiales" de la ciudad de La Plata, se hizo un muestreo de 154 establecimientos educativos, es decir más de un tercio del total. Los resultados son abrumadores: sólo un 1\% de los establecimientos educativos es accesible, un $84 \%$ es inaccesible, y un $15 \%$ no pudo determinarse ya que las 
Zelasqui, I.O. Más allá de una problemática urbana: Accesibilidad edilicia y educación inclusiva para personas con discapacidad. Derecho y Ciencias Sociales. Mayo -Octubre 2021. № 25 .Pgs 100-122. ISSN 1852-2971. Instituto de Cultura Jurídica y Maestría en Sociología Jurídica. Facultad de Ciencias Jurídicas y Sociales. Universidad Nacional de La Plata. Argentina.

autoridades de las escuelas, pese a tener permiso firmado por Inspectora Jefe Distrital de La Plata, se negaron a permitir el acceso al edificio:

\section{Tabla 1 y Gráfico 1: Resultados por variables (valores expresados en absoluto)}

\begin{tabular}{|c|c|c|}
\hline & CUMPLEN & INCUMPLEN \\
\hline ACCESO & 97 & 77 \\
\hline TIMBRE & 14 & 96 \\
\hline AULAS & 89 & 21 \\
\hline BAÑO & 20 & 85 \\
\hline PATIO & 35 & 27 \\
\hline
\end{tabular}

Nota: Las diferencias numéricas de un rubro a otro se deben a la negativa al ingreso en algunos establecimientos o ingresos limitados solo a

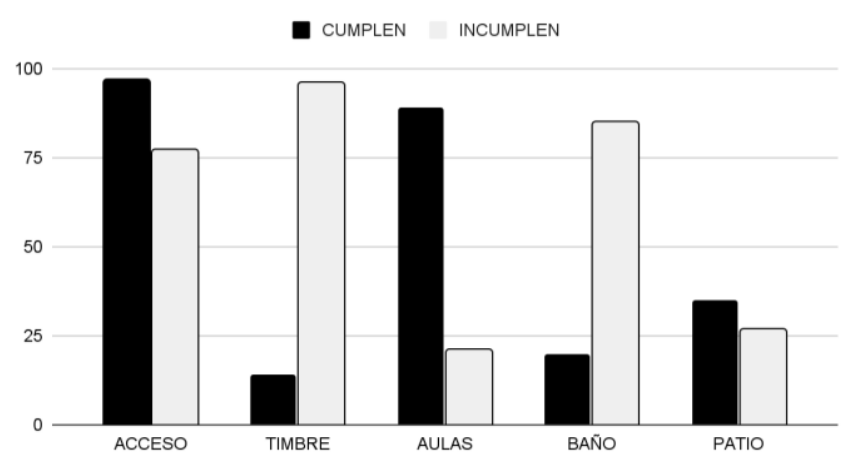
ciertas áreas de la escuela.

Fuente: Elaborado en base a datos obtenidos en relevamiento realizado entre los años 2012 y 2013 en la ciudad de La Plata. Participaron varios actores sociales: integrantes de la Clínica Jurídica de Discapacidad y Derechos Humanos (graduados y estudiantes de derecho), estudiantes de otras carreras (comunicación social y ciencias de la educación), personas con discapacidad, organizaciones sociales y movimientos de PCD.

Consideramos escuela accesible aquella que cumple con estas cinco variables: rampas de acceso, altura de timbre, baño adaptado, amplitud y circulación en aulas y patio. En el mismo sentido, entendemos por escuela no accesible aquella que habiendo observado todas las variables no cumple con alguna de ellas. Con esta categorización y ante las limitaciones mencionadas para medir alguna de las variables, clasificamos los resultados en tres: Escuelas accesibles, no accesibles y sin determinar (porque no se pudo medir alguna de las variables que consideramos para definir la categoría):

Tabla 2 y Gráfico 2: Resultados por categorización de escuelas (valores expresados en porcentaje)

Escuelas Escuelas no Sin accesibles accesibles determinar
- Escuelas accesibles Escuelas no accesibles Escuelas sin determinar $100 \%$

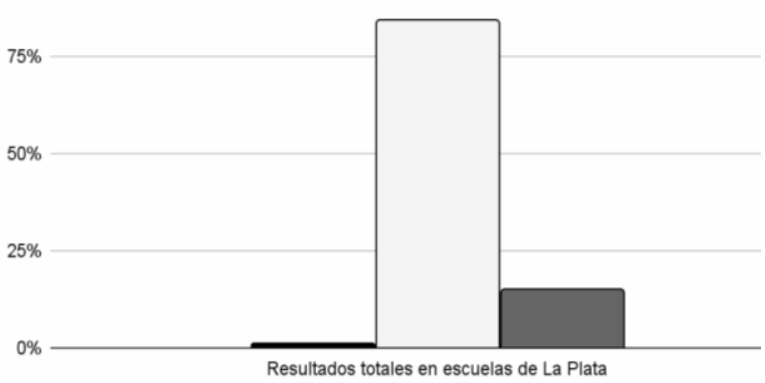


Zelasqui, I.O. Más allá de una problemática urbana: Accesibilidad edilicia y educación inclusiva para personas con discapacidad. Derecho y Ciencias Sociales. Mayo -Octubre 2021. № 25 .Pgs 100-122. ISSN 1852-2971. Instituto de Cultura Jurídica y Maestría en Sociología Jurídica. Facultad de Ciencias Jurídicas y Sociales. Universidad Nacional de La Plata. Argentina.

\begin{tabular}{|c|c|c|c|}
\hline $\begin{array}{l}\text { Resultados } \\
\text { totales } \\
\text { escuelas La } \\
\text { Plata }\end{array}$ & 1\% & $84 \%$ & 15\% \\
\hline \multicolumn{4}{|c|}{$\begin{array}{l}\text { Fuente: Elaborado en base a datos obtenidos en } \\
\text { relevamiento realizado entre los años } 2012 \text { y } 2013 \text { en la } \\
\text { ciudad de La Plata. Participaron varios actores sociales } \\
\text { integrantes de la Clínica Jurídica de Discapacidad y } \\
\text { Derechos Humanos (graduados y estudiantes de derecho) } \\
\text { estudiantes de otras carreras (comunicación social y } \\
\text { ciencias de la educación), personas con discapacidad } \\
\text { organizaciones sociales y movimientos de PCD. }\end{array}$} \\
\hline
\end{tabular}

En consecuencia, producto del relevamiento llevado a cabo en el año 2012/2013, se puede afirmar que la mayoría de las escuelas en la ciudad de La Plata, tanto de gestión pública como privada, son inaccesibles para las personas con discapacidad debido a las múltiples barreras físicas que presentan. Dichas barreras constituyen una de las causas centrales de la exclusión social de niños y niñas con discapacidad de su derecho a la educación inclusiva, reconocida vigorosamente por el art. 24 de la Convención Internacional sobre los Derechos de las Personas con Discapacidad concretando con dicha omisión estructural un verdadero estado de cosas inconstitucional.

Este estado de cosas incompatible con la Constitución y los Tratados Internacionales de Derechos Humanos se genera porque todo el colectivo de niños y niñas con discapacidad motriz de la Ciudad de La Plata deben enfrentarse con fuertes barreras institucionales para hacer efectivo el derecho a la educación, a la inclusión en la comunidad, así como en la formación y preparación para el futuro y que a pesar de haber numerosas leyes que obligan a eliminar las barreras, dichas normativas no son efectivas, no se ven reflejadas en políticas públicas, ni tienen impacto presupuestario.

El presente se trata de un caso que por su complejidad, multiplicidad de derechos violados y afectación a un gran número de personas puede caracterizarse como un caso de perfil “estructural”. En los últimos años el clásico control de constitucionalidad de las normas se ha ido expandiendo con la aparición de los llamados litigios estructurales que, frente a situaciones complejas de violaciones de derechos, requieren de respuestas o medidas creativas para garantizar una tutela judicial efectiva y que los justiciables encuentren amparo en alguno de los poderes del estado.

Los procesos de interés público como el presente, involucran derechos fundamentales colectivos y se caracterizan por ciertas notas típicas: 1) el activismo procedimental y los amplios poderes del juez, tanto en el comando y la dirección del trámite cuanto a la instrucción 
Zelasqui, I.O. Más allá de una problemática urbana: Accesibilidad edilicia y educación inclusiva para personas con discapacidad. Derecho y Ciencias Sociales. Mayo -Octubre 2021. № 25 .Pgs 100-122. ISSN 1852-2971. Instituto de Cultura Jurídica y Maestría en Sociología Jurídica. Facultad de Ciencias Jurídicas y Sociales. Universidad Nacional de La Plata. Argentina.

probatoria y los alcances de la decisión; 2) el método dialogal público que compromete a las partes en la búsqueda de soluciones consensuadas; 3) la función "remedial" dirigida a la modificación "estructural" de las organizaciones burocráticas involucradas y de este modo satisfacer los bienes fundamentales involucrados; 4) una nueva concepción del proceso donde la etapa de ejecución o cumplimiento de la sentencia instituye una continua comunicación entre las partes y el tribunal, anudándose así un vínculo de largo plazo (Berizonce, 2014).

Hasta aquí he presentado la situación en términos de tensión entre derechos humanos (legislación vigente y obligaciones derivadas) y los niveles de vigencia real en materia de políticas públicas sobre accesibilidad en escuelas de La Plata. A continuación, pretendo reflexionar desde otras dimensiones pero en diálogo con lo desarrollado anteriormente. En otras palabras, ir más allá del planteo de una problemática urbana que se vincula con la accesibilidad a la educación inclusiva para personas con discapacidad, sino como elementos que se relacionan y configuran mutuamente.

\section{Distribución espacial del poder e identidades colectivas desterritorializadas.}

Si adoptamos la premisa según la cual los espacios siempre han estado interconectados jerárquicamente en lugar de concebir al espacio como algo inherentemente fragmentado en espacios autónomos, podríamos representar la diferencia a través de la interconexión y superar el ocultamiento de la topografía del poder que presenta a los espacios como naturalmente desconectados (Gupta y Ferguson, 2008). En la distribución espacial del poder, las escuelas comunes participan del sistema de espacios en relación jerárquica con las escuelas especiales y constituyen su identidad en esa relación de diferencia.

Bajo esta perspectiva, el mundo es un conjunto de relaciones de poder que producen diferencias como parte del sistema global de dominación. Nuestra tarea es desnaturalizar las divisiones espaciales que segregan dentro de espacios específicos ("escuelas especiales") para personas que no cumplen con los criterios normalizadores impidiendo el acceso a derechos y la inclusión social. Repensando las relaciones entre poder y espacio se abre la posibilidad de discutir sobre la políticas de acceso edilicio, el derecho a la educación inclusiva, la apropiación de conceptos como inclusión y diversidad, y las percepciones populares sobre las personas con discapacidad. Existe hoy día una esfera pública transnacional que ha posibilitado nuevas formas de identidad que no requieren de la apropiación de espacios estrictamente delimitados. El espacio ha sido re-territorializado obligando a repensar las nociones de comunidad, solidaridad, identidad y diferencia cultural. En el contexto actual de un mundo de identidades desterritorializadas, la 
Zelasqui, I.O. Más allá de una problemática urbana: Accesibilidad edilicia y educación inclusiva para personas con discapacidad. Derecho y Ciencias Sociales. Mayo -Octubre 2021. № 25 .Pgs 100-122. ISSN 1852-2971. Instituto de Cultura Jurídica y Maestría en Sociología Jurídica. Facultad de Ciencias Jurídicas y Sociales. Universidad Nacional de La Plata. Argentina.

cuestión de identidad colectiva cobra un carácter especial (Gupta y Ferguson, 2008). Si bien los autores refieren a las personas refugiadas, migrantes, desplazadas y pueblos sin Estado como los primeros grupos en experimentar estas realidades, reconocen que es una condición que se encuentra generalizada. En ese orden de ideas, la discapacidad también es "algo tan complejo y se experimenta como algo tan desterritorializado...", pues "...se refiere mucho menos a un lugar delimitado que a una condición de ser o posición moral imaginadas" (Gupta y Ferguson, 2008: 240). En ese sentido, considero que el colectivo de personas con discapacidad también se encuadra en una identidad colectiva desterritorializada, la cual no requiere de la apropiación de un lugar delimitado, sino que disputa el espacio en cuanto a su producción de imaginarios, identidades y diferencias, con una perspectiva de universalidad propio del discurso de derechos humanos de evidente carácter transnacional. Si bien es difícil formular movimientos políticos sin referencia a Naciones, se clarifica cuando se radica la causa en un "lugar" y el colectivo se moviliza en torno a los procesos de construcción de ese lugar. Gupta y Ferguson refieren al replanteamiento que algunos autores han hecho respecto de ideas de "hogar" o "campo" para elaborar una crítica feminista o del capitalismo industrial. En este caso el lugar que se pretende replantear es "la escuela" y el proceso de construcción simbólica de ese lugar.

El lugar que ocupan los cuerpos comprende el punto del espacio físico en que un agente está situado, es decir, donde "tiene lugar o existe" tanto como localización o desde una perspectiva relacional, como posición (Bourdieu, 1999). En este sentido, los agentes se configuran como tales en relación con determinados campos sociales, se sitúan en un lugar del espacio social caracterizado por su posición relativa con respecto a los otros lugares y por la distancia que los separa. Entonces, siguiendo con Bourdieu, el espacio social se define por la exclusión o distinción mutua de las posiciones que lo constituyen:

“Así, la estructura del espacio se manifiesta, en los contextos más diversos, bajo la forma de oposiciones espaciales donde el espacio habitado (o apropiado) funciona como una especie de simbolización espontánea del espacio social. En una sociedad jerarquizada no hay espacio que no esté jerarquizado y no exprese las jerarquías y las distancias sociales, de un modo (más o menos) deformado y sobre todo enmascarado por el efecto de naturalización que entraña la inscripción duradera de las realidades sociales en el mundo natural: así, 
Zelasqui, I.O. Más allá de una problemática urbana: Accesibilidad edilicia y educación inclusiva para personas con discapacidad. Derecho y Ciencias Sociales. Mayo -Octubre 2021. № 25 .Pgs 100-122. ISSN 1852-2971. Instituto de Cultura Jurídica y Maestría en Sociología Jurídica. Facultad de Ciencias Jurídicas y Sociales. Universidad Nacional de La Plata. Argentina.

determinadas diferencias producidas por la lógica histórica pueden parecer como surgidas de la naturaleza de las cosas..." (Bourdieu, 1999: 120).

En este marco la distinción entre "escuela común o normal” y "escuela especial" es la traducción del espacio social en el espacio físico, dicho de otro modo, una proyección espacial de la diferencia social entre los cuerpos. El poder sobre determinado espacio físico apropiado, en este caso las escuelas, se manifiesta en la relación entre la estructura espacial de distribución de los agentes y la de los bienes y servicios. En este sentido, el acceso a un bien fundamental como la educación, está determinado por la relación entre ésta y la persona o cuerpo "común o normal". Las estructuras de poder del espacio social y su inercia histórica se inscribe en el espacio físico generando barreras y expulsando a toda persona que no cumpla con dichos criterios normalizadores. El espacio social reificado distribuye en el espacio físico diferentes bienes y servicios, también a diferentes agentes o grupos provistos de oportunidades, más o menos efectivas, de apropiación de esos bienes y servicios (Bourdieu, 1999).

Si bien excede al presente trabajo (que se centra en el acceso físico) cabe resaltar, para una mejor comprensión de la problemática, las implicancias en términos educativos y sociales que supone la distinción entre escuela común y especial, así como los efectos del tránsito por una u otra. Tal como desarrollé al referirme al derecho a la educación inclusiva, éste es precondición de otros derechos; en especial por la relación entre el derecho a la educación, el trabajo, la cultura y el medio social, al incidir sobre la formación para el futuro y facilitar la autonomía posibilitando el diseño del propio plan de vida. A su vez, repercute en la deliberación democrática, posibilitando vínculos en la comunidad basados en la diversidad.

En este orden de ideas, la escuela "común" es el lugar de la educación, es decir, el espacio físico que concentra los polos positivos del campo y a la mayoría de los agentes que ocupan posiciones dominantes, poder que les permite apropiarse de dicho espacio. Pensada relacionalmente, la escuela "especial" es la privación de la escuela "común" (en términos de integración) y consecuentemente de la educación (inclusiva). Es entonces, el espacio físico que concentra los polos negativos del campo y a la mayoría de los agentes que ocupan posiciones de discapacidad.

Estas oposiciones sociales físicamente objetivadas, tienden a reproducirse en el pensamiento y el lenguaje, convirtiéndose progresivamente en estructuras mentales, categorías de percepción y apreciación o en sistemas de preferencias (normal/común/especial/incapaz, etc): 
Zelasqui, I.O. Más allá de una problemática urbana: Accesibilidad edilicia y educación inclusiva para personas con discapacidad. Derecho y Ciencias Sociales. Mayo -Octubre 2021. № 25 .Pgs 100-122. ISSN 1852-2971. Instituto de Cultura Jurídica y Maestría en Sociología Jurídica. Facultad de Ciencias Jurídicas y Sociales. Universidad Nacional de La Plata. Argentina.

"Debido al hecho de que el espacio social está inscrito simultáneamente en las estructuras espaciales y en las estructuras mentales que son parte del producto de incorporación de las primeras, el espacio es uno de los lugares donde se afirma y ejerce el poder, sin duda bajo la forma más sutil, la de la violencia simbólica como violencia inadvertida: los espacios arquitectónicos cuyas conminaciones mudas interpelan directamente al cuerpo -y obtienen de éste, con tanta infalibilidad como la etiqueta en las sociedades cortesanas, la reverencia, el respeto que nace del alejamiento o, mejor, del estar lejos, a distancia respetuosa-, son sin duda los componentes fundamentales, a causa de su misma invisibilidad (...), del simbolismo del poder y de los efectos totalmente reales del poder simbólico.” (Bourdieu, 1999: 122).

Si la capacidad de dominar el espacio y apropiarse de los bienes que en él se distribuyen depende del capital que se tiene, en el caso de las escuelas, el capital que se requiere para acceder al bien educación es un cuerpo o persona que se corresponda con los criterios de normalidad socialmente instituidos. De este modo, quienes carecen de ese capital, son mantenidos a distancia (física o simbólicamente) del acceso a ese bien, así como a quienes detentan el capital les permite, además de mantener a distancia a las personas indeseables, garantizar la proximidad con el bien deseado facilitando, a su vez, la acumulación y reproducción de dicho capital.

Como consecuencia de esta relación entre espacio y bienes surgen las luchas por la apropiación del espacio, las cuales pueden asumir forma individual o colectiva. La primera se daría en los supuestos que Bourdieu denomina desplazamientos o traslados, donde el acercamiento espacial no garantiza el acercamiento social. La ocupación legítima del lugar supone el capital antes referido, por tanto, se puede ocupar físicamente el lugar pero sin habitarlo, tal como sucede en los supuestos de integración educativa (sin inclusión). Desde esta lógica quienes entran al espacio deben cumplir las condiciones que este exige tácitamente a sus ocupantes para no sentirse desplazados, experimentando una sensación de exclusión capaz de privarlo de la educación por no presentar todos los rasgos deseados o presentar (al menos) una de las propiedades indeseables. 
Zelasqui, I.O. Más allá de una problemática urbana: Accesibilidad edilicia y educación inclusiva para personas con discapacidad. Derecho y Ciencias Sociales. Mayo -Octubre 2021. № 25 .Pgs 100-122. ISSN 1852-2971. Instituto de Cultura Jurídica y Maestría en Sociología Jurídica. Facultad de Ciencias Jurídicas y Sociales. Universidad Nacional de La Plata. Argentina.

La forma colectiva de la lucha por el espacio tiene crucialmente por objeto la política del Estado, que contiene un inmenso poder sobre el espacio derivado de su capacidad para decidir sobre el mercado del suelo, la vivienda y también, del trabajo y la escuela. (Bourdieu, 1999).

\section{Una estrategia colectiva de lucha por el espacio: resistencias, disputas y contra-espacios.}

La noción de identidad colectiva desterritorializada pero, a su vez, situada en un "lugar" como proceso de construcción de imaginarios, identidades y diferencias, puede articularse con la distinción entre movimientos socioespaciales y movimientos socioterritoriales que elabora Bernardo Fernandes Mançano. Partiendo de la noción de movimiento social propia de la sociología, el autor pretende pensar más allá de las formas, acciones y relaciones de los mismos, buscando comprender los espacios y territorios producidos o construidos por los movimientos. Todos los movimientos producen algún espacio, pero no todos los movimientos tienen el territorio como objetivo. La diferencia entre ambos radica justamente en que los movimientos socioterritoriales tienen el territorio no solamente como objeto, sino que éste es esencial para su existencia (movimientos campesinos, indígenas, entre otros). Los movimientos socioespaciales, en cambio, son una agencia de mediación y representaciones, sujetos reivindicando espacios, agencias intermediarias que producen espacios políticos y se espacializan (Fernandes, 2005). Es decir, trabajan con representaciones de intereses pero sin poseer un territorio definido, creando relaciones sociales para tratar directamente sus intereses y así producir sus propios espacios y territorios o modificar los existentes. En este sentido, al hacer partícipe a la Clínica Jurídica de Derechos Humanos y Discapacidad (UNLP), las personas con discapacidad pretende generar ámbitos de relación con el Estado con miras a crear/modificar el espacio, en este caso, generar escuelas accesibles. No podrían caracterizarse como movimiento socioterritorial porque aun si pensáramos conceptualmente a las escuelas de La Plata como un posible territorio, el colectivo, su identidad e intereses lo exceden. Dicho de otro modo, esta agencia de mediación representa los intereses y derechos de las PCD en general, no se agota en la accesibilidad a las escuelas. Además, pensando específicamente el derecho a la educación inclusiva, si bien está ligado a la accesibilidad edilicia de las escuelas, esto sólo no alcanza para garantizarlo (hay que repensar proyectos pedagógicos, asistentes personales, etc). Tal como señala Agustina Palacios "una educación inclusiva no es una cuestión tan simple como la modificación de la organización de la escuela, sino que implica un cambio en la ética de la escuela. No se requiere simplemente que los maestros adquieran nuevas habilidades, sino que se necesita asimismo un compromiso. No alcanza con la aceptación de la diferencia, sino 
Zelasqui, I.O. Más allá de una problemática urbana: Accesibilidad edilicia y educación inclusiva para personas con discapacidad. Derecho y Ciencias Sociales. Mayo -Octubre 2021. № 25 .Pgs 100-122. ISSN 1852-2971. Instituto de Cultura Jurídica y Maestría en Sociología Jurídica. Facultad de Ciencias Jurídicas y Sociales. Universidad Nacional de La Plata. Argentina.

que se requiere una valoración de la diferencia. Lo que se necesita, en definitiva, es un compromiso moral con la inclusión de todas las personas dentro de un sistema educativo, como parte de un compromiso más amplio que aspira a la inclusión de todas las personas dentro de la sociedad." (Palacios, 2008: 132) En síntesis, los proyectos de extensión universitaria como el mencionado, dedicado a las problemáticas de las PCD mediante una perspectiva de ddhh, establece lazos con la comunidad y genera un espacio de participación para esta minoría históricamente invisibilizada. Recibiendo sus demandas y generando por medio del litigio estructural un ámbito de mediación entre ellas y el Estado para la concreción y construcción colectiva de políticas públicas acordes a los DDHH y compromisos asumidos por el Estado que garanticen a las PCD el ejercicio de sus derechos con libertad y autonomía para desarrollar su proyecto de vida en igualdad de condiciones, afianzando su dignidad como persona. Mediante esta estrategia, el movimiento social de PCD lleva adelante una forma colectiva de lucha por el espacio.

I) Políticas públicas en accesibilidad educativa: efectos, relaciones y sistemas de pensamiento. Conforme he desarrollado, al cotejar la vasta normativa legal, constitucional/convencional y su correlato fáctico con la situación edilicia de las escuelas en La Plata, queda en evidencia que dicho plexo normativo no garantizan ni se reflejan de modo automático en la formulación y ejecución de las políticas públicas en la materia. Como se ha dicho, basta con realizar un relevamiento para observar los desfasajes existentes. Es necesario, entonces, poner de manifiesto estos escenarios donde el contexto normativo institucional da una apariencia acorde a los derechos y parámetros establecido por el sistema internacional de derechos humanos. Al poner de relieve la situación real de las escuelas, evidenciamos el resultado de las políticas públicas en la materia, el cual se traduce en escuelas accesibles o inaccesibles, impactando esto en el reconocimiento al derecho humano a una educación inclusiva de niños, niñas y adolescentes. El contexto fáctico observado podría suponer una situación de segregación, la cual se da cuando la división social del espacio es el resultado de la aplicación de políticas o prácticas de exclusión de ciertos grupos respecto de espacios específicos, es decir, cuando existen prácticas activas de segregación espacial (Duahu, 2013). Utilizando esta conceptualización sólo a escala de la ciudad de La Plata y sus escuelas, se puede afirmar que las políticas públicas educativas han estado orientadas hacia la exclusión de las PCD, relegándolas a otro espacio específico, las escuelas denominadas "especiales", vedándoles su derecho a una educación inclusiva en igualdad de condiciones con el resto. La segregación 
Zelasqui, I.O. Más allá de una problemática urbana: Accesibilidad edilicia y educación inclusiva para personas con discapacidad. Derecho y Ciencias Sociales. Mayo -Octubre 2021. № 25 .Pgs 100-122. ISSN 1852-2971. Instituto de Cultura Jurídica y Maestría en Sociología Jurídica. Facultad de Ciencias Jurídicas y Sociales. Universidad Nacional de La Plata. Argentina.

descripta es el resultado de la formulación de políticas públicas en el marco institucional tradicional, funcionando como un campo de actividad confinado exclusivamente en las elites gubernamentales sin participación de la ciudadanía, siendo las políticas un programa por medio de los cuales los funcionarios del Estado intentan gobernar. En este entendimiento las políticas públicas son instrumentos de esta ambición autoritaria, enmarcado dentro de las teorías de elección racional y en los modelos positivistas, donde los actores persiguen metas con un norte calculando costos y beneficios (Duhau, 2013).

La esfera ideal-normativa debe plasmarse por medio de las políticas públicas en un escenario concreto de escuelas accesibles para garantizar el derecho humano a la educación (inclusiva). Es en el plano material donde se pone de relieve la interpretación y entendimiento que de los novedosos principios jurídicos hacen los agentes que formulan y ejecutan las políticas públicas. Es decir, el escenario real de las escuelas producto de las políticas implementadas manifiesta el imaginario que el poder político tiene respecto de las personas con discapacidad. Las políticas pueden ser interpretadas en cuanto a sus efectos (lo que producen), las relaciones que crean y los sistemas de pensamientos más amplios en medio de los cuales están inmersas (Shore, 2010). En el supuesto de análisis, las políticas públicas resisten la generación de escuelas accesibles ediliciamente, en consecuencia, se oponen al ideario de inclusión educativa y diversidad en cualquier escuela. En efecto, refuerzan y reproducen relaciones de poder, caracterizada por la opresión y segregación de las personas con discapacidad, a quienes se les asigna categorías disvaliosas y roles sociales subalternos.

Sumado a ello, el uso del discurso en boga en la normativa vigente funciona para disfrazar, invisibilizar y por ende naturalizar este ideario sobre las personas con discapacidad. En esta lógica, la consagración de los derechos en el plano ideal-normativo actúa como herramienta de control social que sostiene pautas histórico culturales e institucionales que el nuevo modelo pretende superar para incluir a la ciudadanía plena a las personas con discapacidad.

Para superar la maniobra descripta y retomando el punto referente al litigio estructural, este tipo de proceso con las características innovadoras descritas por Berizonce, tiene la particularidad de darse en un contexto fáctico evidentemente inconstitucional que afecta a un amplio grupo de personas. En consecuencia, no se pone en discusión el derecho ya que resulta ostensible que las personas con discapacidad tienen derecho a infraestructuras edilicias accesibles. Más aún, en el caso de los establecimientos educativos, difícilmente alguien pueda poner en duda que los niños, niñas y adolescentes con discapacidad tengan derecho a una educación inclusiva. Procesos judiciales como éstos, abren un ámbito de discusión respecto de 
Zelasqui, I.O. Más allá de una problemática urbana: Accesibilidad edilicia y educación inclusiva para personas con discapacidad. Derecho y Ciencias Sociales. Mayo -Octubre 2021. № 25 .Pgs 100-122. ISSN 1852-2971. Instituto de Cultura Jurídica y Maestría en Sociología Jurídica. Facultad de Ciencias Jurídicas y Sociales. Universidad Nacional de La Plata. Argentina.

cómo debe ser la política pública para ser acorde a los requerimientos de las personas y estándares de ddhh (en este caso, cuándo una escuela se considera accesible), y se establece un plan de cumplimiento o ejecución de la misma. Es decir, se formula la política pública conjuntamente con los interesados y se ejecuta en el ámbito judicial, por lo tanto, con una autoridad de control con el poder suficiente para auditar el accionar de la administración pública en la ejecución del compromiso asumido, conforme los plazos y parámetros planteados y acordados.

\section{II) Resistencias, disputas y contra-espacios.}

Sobre la comprensión del espacio en torno a lo político, saturado por una red compleja de relaciones de poder/saber que se expresan en paisajes materiales y discursos de dominación, pueden presentarse también formas de "espacialidad de resistencia" (Oslender, 2002). Los obstáculos que presentan las estructuras en su interacción con los actores sociales, también pueden ser ajustadas, cambiadas o inclusive derrotadas por los mismos actores. Las prácticas sociales pueden entonces reproducirlas o resistirse a ellas, siendo el espacio un sitio conflictivo donde se disputa el uso que se le da al mismo entre dominación y resistencia, donde los movimientos sociales articulan y median en esas luchas. En este entendimiento, la identidad colectiva del movimiento y sus acciones se articulan y construyen físicamente, es decir, por la comprensión que se tiene de ese espacio. Su lucha es por el espacio material y sus interpretaciones y representaciones. La accesibilidad física o edilicia significa entonces una lucha también por una reinterpretación del espacio escuela y la educación, en términos inclusivos y de diversidad.

Para comprender esta espacialidad de resistencia es necesario retomar el trabajo sobre producción del espacio de Henri Lefebvre en sus tres momentos (prácticas espaciales, representaciones del espacio y espacio de representación) estableciendo una interdependencia y relación dialéctica entre lo percibido, lo concebido y lo vivido (Oslender, 2002).

Las prácticas espaciales refieren a un proceso histórico de burocratización de las formas en que generamos, utilizamos y percibimos el espacio, las cuales sedimentan un "espacio concreto". Las representaciones del espacio suponen aquellos saberes técnicos y racionales (científicos/as, urbanistas, ingenieros/as, etc) mediante los cuales se "explican" o "entienden" los espacios concebidos. Estos saberes se vinculan al poder dominante y las representaciones normalizadas generadas por una "lógica de visualización" hegemónica que hace "legible" el espacio mediante una simplificación del mismo. Una visión normalizada que ignora luchas, 
Zelasqui, I.O. Más allá de una problemática urbana: Accesibilidad edilicia y educación inclusiva para personas con discapacidad. Derecho y Ciencias Sociales. Mayo -Octubre 2021. № 25 .Pgs 100-122. ISSN 1852-2971. Instituto de Cultura Jurídica y Maestría en Sociología Jurídica. Facultad de Ciencias Jurídicas y Sociales. Universidad Nacional de La Plata. Argentina.

ambigüedades, y otras formas de ver, percibir e imaginar el mundo (Oslender, 2002). Tal como señala el autor, su efecto es de abstracción y descorporealización del espacio, siempre apoyado por argumentos científicos y apelando a una "verdadera" representación. De este modo, en la medida que “...el espacio abstracto tiende hacia la homogeneización, hacia la eliminación de diferencias o peculiaridades existentes, un nuevo espacio solamente puede surgir (o producirse) si se acentúan diferencias" (Lefebvre, 1974: 110). Para ello toman un rol central los espacios de representación, aquellos espacios vividos que representan modos de conocimiento no hegemónicos, informales o subalternos, arraigados en experiencias saturadas de significados, articulados históricamente de modo flexible y dinámico. Estos espacios de representación evidencian las contradicciones del espacio abstracto y conducen, como consecuencia, a la búsqueda de un "espacio diferenciado" o "contra-espacio" donde se articulan las diversas resistencias como una política sobre el espacio. Estos tres momentos están interconectados, son interdependientes, contemporáneos y se relaciona dialécticamente (Oslender, 2002). Es decir, el escenario del espacio de representación es aquel dominado por las representaciones normalizadoras al cual se resiste, buscando apropiarse del imaginario propio de las representaciones dominantes para resultar en un nuevo espacio.

\section{Conclusiones}

Desde la cosmovisión del modelo social de ddhh que pone el foco en la diversidad e inclusión, las escuelas "comunes" o "normales" deben garantizar el acceso, permanencia y egreso de toda persona sin distinción. Este paradigma que incluye a corporalidades disidentes en las "escuelas comunes" pone en evidencia las contradicciones de ese espacio abstracto normalizado. Esas contradicciones puestas de relieve podrían traccionar una política de educación inclusiva que genere un "espacio diferenciado", dicho de otro modo, un espacio inclusivo que contemple la diferencia y diversidad de toda persona. En consecuencia, para la implementación del modelo social de ddhh, es menester pensar y disputar las políticas que permitan primero el acceso para facilitar luego la generación de ese nuevo espacio.

La concepción tradicional en la formulación de las políticas públicas no basta para cumplimentar derechos humanos fundamentales, aun cuando éstos se encuentran consagrados normativamente, se requiere de una continua disputa para su consolidación por medio de políticas que los efectivicen. Frente al panorama de inaccesibilidad en que concluyó el relevamiento en establecimientos educativos de la ciudad de la plata (solo el $1 \%$ sobre 154 establecimientos educativos son accesibles), el litigio estructural puede facilitar un ámbito de 
Zelasqui, I.O. Más allá de una problemática urbana: Accesibilidad edilicia y educación inclusiva para personas con discapacidad. Derecho y Ciencias Sociales. Mayo -Octubre 2021. № 25 .Pgs 100-122. ISSN 1852-2971. Instituto de Cultura Jurídica y Maestría en Sociología Jurídica. Facultad de Ciencias Jurídicas y Sociales. Universidad Nacional de La Plata. Argentina.

diálogo y construcción colectiva de las políticas públicas. Este espacio de mediación permite articular una formulación conjunta de políticas públicas con perspectiva de derechos humanos y participación de los sujetos involucrados en las misma. Además, cuenta con un seguimiento garantizado por el control del poder jurisdiccional, permitiendo de este modo eludir la fachada normativa de reconocimiento de derecho frente un escenario fáctico de flagrante incumplimiento y violación de derechos humanos fundamentales.

En suma, el litigio estructural puede servir como estrategia de disputa sobre el espacio facilitando un ámbito de participación y formulación conjunta de las políticas públicas y de control en la ejecución de las mismas. Este marco conceptual es extensible a otros supuestos de violación de derechos humanos que afectan a grandes grupos en situación de vulnerabilidad de manera generalizada, siempre que se den ciertos requisitos. Por un lado, el activismo, organización y movilización de dichas minorías, herramientas necesarias para disputar, apropiarse y modificar esos espacios. Por otra parte, una concepción del espacio en torno a lo político, es decir, que cuestione los espacios entendidos como algo naturalmente dado y distribuidos neutralmente. Resulta imperioso dar cuenta de la distribución espacial del poder, los procesos de construcción de esos lugares y sus efectos respecto de las personas. La lucha colectiva por la apropiación del espacio consiste justamente en desentrañar las representaciones e imaginarios sociales que fundan esos espacios, su distribución y características para desmontar las relaciones de poder en que se sustentan y reproducen. De este modo podrán generar nuevos espacios o "contra-espacios" que faciliten otros modos de vinculación, en este caso, inclusivos en la diversidad.

Para finalizar, las relaciones establecidas a lo largo de este trabajo entre la accesibilidad en establecimientos educativos desde una perspectiva de ddh con la accesibilidad edilicia como problemática urbana, pensadas interconectadas y relacionadas dialécticamente abren diversas dimensiones de trabajo posibles sobre esta problemática. Solo hemos presentado algunos interrogantes y líneas preliminares de análisis, cada una de las cuales, requiere mayor profundización para desplegar su complejidad y potencialidad, invitando a su indagación en trabajos futuros.

\section{Bibliografía}

Berizonce, R.O. (2014). "Tutelas Procesales Diferenciadas como Proyección del Movimiento de Acceso a la Justicia". En la obra colectiva Tutelas procesales diferenciadas de los derechos económicos, sociales y culturales, p. 19 y ss. La Plata: FCJS-UNLP. 
Zelasqui, I.O. Más allá de una problemática urbana: Accesibilidad edilicia y educación inclusiva para personas con discapacidad. Derecho y Ciencias Sociales. Mayo -Octubre 2021. № 25 .Pgs 100-122. ISSN 1852-2971. Instituto de Cultura Jurídica y Maestría en Sociología Jurídica. Facultad de Ciencias Jurídicas y Sociales. Universidad Nacional de La Plata. Argentina.

Bourdieu, P. (1999). "Efectos del lugar”. En La miseria del mundo, pp. 119-124. Madrid: Akal ediciones.

Duhau, E. (2013). La división social del espacio metropolitano. Una propuesta de análisis. Buenos Aires: Revista Nueva Sociedad No 243: 79-91.

Fernándes Mançano, B. (2005). Movimientos socioterritoriales y movimientos socioespaciales. Contribución teórica para una lectura geográfica de los movimientos sociales. Buenos Aires: Revista OSAL n 16, enero-abril 2005, pp. 273-283.

Gupta, A. y Ferguson, J. (2008). Más allá de la cultura: Espacio, identidad y las políticas de la diferencia. Bogotá: revista ANTÍPODA No 7, Julio-Diciembre 2008, pp. 233-256.

Lefebvre, H. (1974). La Producción del espacio. Madrid: Capitán Swing ediciones, 2013.

Oslender, U. (2002). Espacio, lugar y movimientos sociales: hacia una "espacialidad de resistencia". Barcelona: Revista Scripta Nova, Universidad de Barcelona. Vol. VI, núm. 115.

Palacios, A. (2008). El modelo social de discapacidad: orígenes, caracterización y plasmación en la Convención Internacional sobre los Derechos de las Personas con Discapacidad. Madrid: CERMI ediciones.

Shore, C. (2010). La antropología y el estudio de la política pública: reflexiones sobre la “formulación" de las políticas. Bogotá: Antípoda, revista de Antropología y Arqueología $\mathrm{N}^{\mathrm{o}} 10$, enero/junio 2010, pp. 21-49.

\section{Documentos internacionales y normativa local referenciadas.}

-Convención Internacional Sobre los Derechos de las Personas con Discapacidad. Nueva York: 13 de Diciembre de 2006, por resolución 61/106 en Asamblea General de Naciones Unidas. Disponible en: https://www.un.org/esa/socdev/enable/documents/tccconvs.pdf

-Declaración Universal de Derechos Humanos. París: 10 de diciembre de 1948, por resolución 217 A (III) Asamblea General de Naciones. Disponible en: https://www.ohchr.org/EN/UDHR/Documents/UDHR_Translations/spn.pdf

-Pacto Internacional de Derechos Civiles y Políticos. Nueva York: 16 de Diciembre de 1966, por resolución 2200 A (XXI) en Asamblea General de Naciones Unidas. Disponible en: https://www.ohchr.org/sp/professionalinterest/pages/ccpr.aspx

-Pacto Internacional de Derechos Económicos, Sociales y Culturales. Nueva York: 16 de diciembre de 1966, por resolución 2200 A (XXI) en Asamblea General de Naciones Unidas. Disponible en: https://www.ohchr.org/sp/professionalinterest/pages/cescr.aspx

-Convención Interamericana para la Eliminación de Todas las Formas de Discriminación contra las Personas con Discapacidad. Guatemala: 8 de Junio de 1999, por resolucion A-65 en Asamblea General de la Organizacion de los Estados Americanos: Disponible en: https://www.oas.org/juridico/spanish/tratados/a-65.html

-Declaración Americana de los Derechos y Deberes del Hombre. Bogotá: 30 de Abril de 1948, aprobada en la IX Conferencia Internacional Americana, Organización de los Estados Americanos.

-Convención Americana sobre Derechos Humanos. San José de Costa Rica: 22 de de Noviembre de 1969, por resolución B-32 en Asamblea General de la Organización de los 
Zelasqui, I.O. Más allá de una problemática urbana: Accesibilidad edilicia y educación inclusiva para personas con discapacidad. Derecho y Ciencias Sociales. Mayo -Octubre 2021. № 25 .Pgs 100-122. ISSN 1852-2971. Instituto de Cultura Jurídica y Maestría en Sociología Jurídica. Facultad de Ciencias Jurídicas y Sociales. Universidad Nacional de La Plata. Argentina.

Estados Americanos: Disponible en: https://www.oas.org/dil/esp/tratados_b32_convencion_americana_sobre_derechos_humanos.htm

-Declaración de Salamanca y Marco de Acción para las Necesidades Educativas Especiales. Salamanca, España: 7-10 de Junio de 1994, aprobada en conferencia Mundial sobre necesidades educativas especiales: Acceso y Calidad (UNESCO). Disponible en: https://www.unioviedo.es/ONEO/wp-content/uploads/2017/09/Declaraci\%C3\%B3nSalamanca.pdf

-Constitución de la Nación Argentina. Sancionada en 1853 con las reformas de los años 1860, 1866, 1898, 1957 y $1994 . \quad$ Disponible en: http://servicios.infoleg.gob.ar/infolegInternet/anexos/0-4999/804/norma.htm

-Ley nacional 24.314 "Sistema de Protección Integral de los discapacitados. Accesibilidad de personas con movilidad reducida". Sancionada en Marzo 15 de 1994. Disponible: http://servicios.infoleg.gob.ar/infolegInternet/anexos/0-4999/713/norma.htm

-Ley provincial 10.592 "Régimen Jurídico Básico e Integral para las Personas Discapacitadas”. Sancionada en Octubre 22 de 1987. Disponible actualizada en: https://normas.gba.gob.ar/documentos/B1eGmU4B.html

-Decreto 1149/90. Reglamentario de Ley 10.592, 6 de Abril de 1990. Disponible en: https://normas.gba.gob.ar/documentos/VwvNW4CW.html

-Ley provincial 13.688 "Ley de educación provincial". Sancionada en Junio 27 de 2007. Disponible en: https://normas.gba.gob.ar/documentos/B15nYIQV.html 